\title{
MESO-SCALE COMPUTATIONAL MODELING OF HYPERVELOCITY IMPACT DAMAGE IN ADVANCED MATERIALS
}

\author{
Aleksandr Cherniaev \\ Department of Mechanical, Automotive and Materials \\ Engineering \\ University of Windsor \\ Windsor, Canada
}

\author{
Igor Telichev \\ Department of Mechanical Engineering \\ University of Manitoba \\ Winnipeg, Canada
}

\begin{abstract}
Modeling of hypervelocity impact (HVI) on materials and structures is often associated with high computational expenses, especially when inhomogeneous materials are involved. To reduce computational cost, complex materials are often represented in modeling as homogeneous substances with the effective properties similar to those of the real materials. Although this approach has been successfully used in modeling of HVI on different materials with complex architecture, there are applications where it may not be applicable due to significant influence of materials' mesoscale features on resulting HVI damage. Two of such applications are considered in this study, and include simulation of HVI on sandwich panels with metallic foamcores, and composites fabricated by filament winding. In the former case, adequate modeling of the multi-shock action of the foam ligaments on hypervelocity fragment cloud propagating through the foam core requires an explicit representation of the foam geometry in numerical model. In the latter case, the meso-scale modeling is required due to experimentally observed dependence of HVI damage of the composite on the particular filament winding pattern used in its fabrication. The study presents numerical models developed for both of these applications and compares numerical results with obtained experimental data.
\end{abstract}

\section{Hypervelocity impact, orbital debris, advanced materials}

\section{INTRODUCTION (HEADING 1)}

Models for the simulation of impact behavior of different inhomogeneous materials have been described in the literature and implemented in commercial software packages, such as Autodyn, LS-Dyna etc. These models are often based on the homogenization of advanced materials with complex microand meso-structure, and their representation in the modeling as a macroscopically homogeneous media with effective properties equivalent to those of the real materials. Although computationally efficient, this approach may be too simplistic for some materials with meso-scale inhomogeneities. This paper considers two problems, where simulation of damage of inhomogeneous materials required meso-scale representation of the materials' architecture. These examples are modeling of hypervelocity impact on a foam-core sandwich panel and simulation of HVI-induced damage in CFRP composites fabricated by filament winding.

Open-cell foam-core sandwich panels are considered by many researchers as a promising alternative to honeycomb-core panels in MMOD protection applications. For instance, in an experimental study presented by NASA [1], foam-core sandwich panels (FCSPs) with 25-30\% lower areal density than HCSPs demonstrated better ballistic performance. In [2], it was determined that, depending on impact conditions, doublelayer foam shields provided from 3 to $15 \%$ improvement in critical projectile diameter as compared to double-layer honeycomb shields of similar weight. The performance improvement in case of using the foams was not explained only as a result of the simple absence of cells that "channel" the fragment cloud. Rather, the radial expansion of fragment cloud and repeated impact of fragments on individual foam cell ligaments induced further fragmentation, melt, and vaporization of fragments [3].

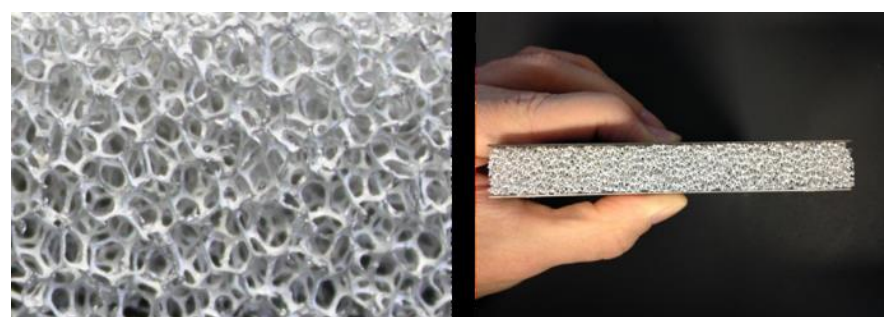

Figure 1. Open-cell aluminum foam (left) and a fragment of a foam-core sandwich panel (right)

Material models implemented in commercial hydrocodes and recommended for simulation of impact on foams are represented, for example, by the LS-DYNA's *MAT_057 (*MAT_LOW_DENSITY_FOAM), *MAT_063 (*MAT_CRUSHABLE_FOAM), and a set of equations of state for porous materials in AUTODYN (Crushable foam EOS, Compaction EOS, P-Alpha EOS). These and other available material models treat the foams as homogeneous materials and, therefore, a priori unable to represent multi- 
shock interactions of foam ligaments with fragments of debris cloud propagating through the foam.

Composite fabricated by filament winding is another example of a material with the meso-scale inhomogeneity. This feature results from multiple interweavings of filament bands forming a filament-wound composite part. In space, these materials can be used in spacecraft propellant tanks (so-called composite overwrapped pressure vessels) and remote manipulator systems (e.g., Canadarm). Behavior of composites under HVI has been studied experimentally and numerically by many researchers, e.g. [4, 5]. However, most of the reported work was confined to the standard laminated composites and homogenization-based simulation techniques, whereas less attention has been paid to the filament-wound materials.

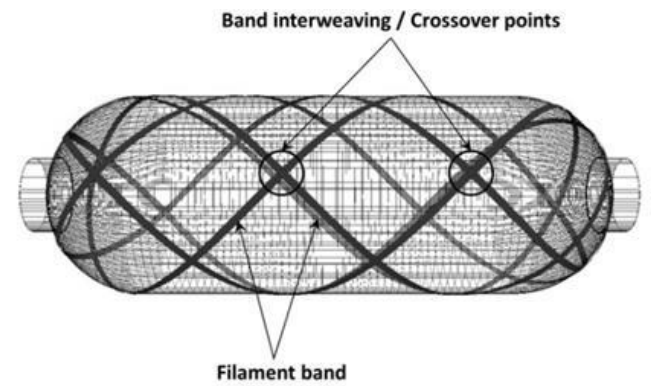

Figure 2. Filament winding

In filament winding, the carriage unit of a winding machine moves back and forth relative to the rotating mandrel and lays down on it the resin-impregnated fibers (towpreg) at a specific angle. Fibers are placed onto a mandrel in the form of filament bands, each containing few thousands of fibers. Fig. 2 illustrates the initial stages of filament winding. At each circuit (one back and forth pass of the carriage unit along a mandrel), filament bands are laid on a mandrel at "+" (forward motion) and "-" (back motion) angle. Each circuit results in crossovers between filament bands of "+" and "-" angle. The presence of crossovers is intrinsic to filament winding and forms its well recognizable patterns. The choice of a particular winding pattern is often up to a manufacturing engineer, as different patterns can be easily programmed using software supplied with the filament winding equipment (Fig. 3).
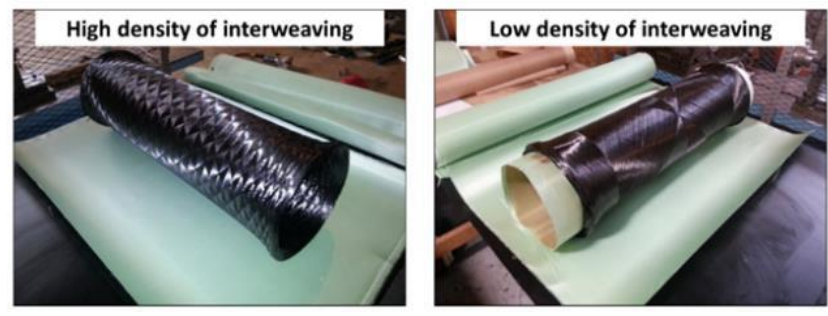

Figure 3. CFRP weaves of different winding patterns

Also, filament-wound composites contain two types of voids: voids internal to filament bands, and voids at the filament bands' crossover regions (Fig. 4). Voids of the latter type represent additional meso-scale feature of filament-wound composites. It is believed that they may result in stress concentration at crossovers leading to formation of cracks and preliminary disintegration of the filament wound composite when subjected to static or impact loading.
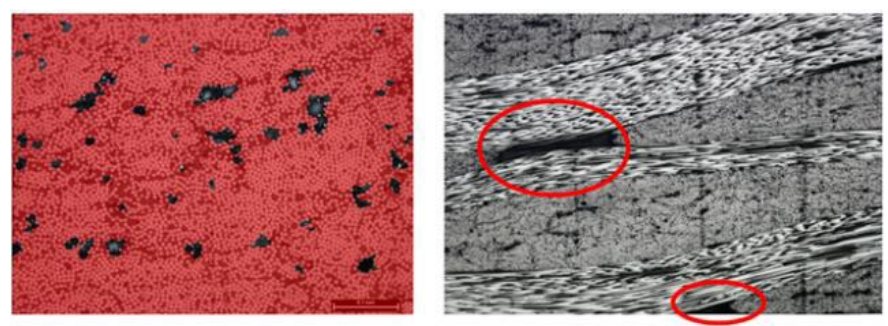

Figure 4. Voids in the filament-wound composite: intra-band voids (left) and voids at the crossovers (right)

\section{MODELING OF OPEN-CELL FOAM}

A mesoscale approach to the modeling of open-cell foam geometry must be used in the HVI simulations to represent multi-shock interactions of the foam ligaments and hypervelocity fragment cloud propagating through the foam. The original structure that forms metallic foam is a threedimensional array of bubbles having a maximum volume for the minimal surface area and surface energy. During the fabrication process, membranes of the bubbles are being removed, leaving an interconnected network of solid struts. A common approach to geometric modeling of open-cell foams is based on Wearie-Phelan idealization [6]. It represents a structure consisting of equal-volume bubbles of two different shapes: namely, the 14-sided tetrakaidecahedron (Fig. 5a) and the 12-sided dodecahedron (Fig. 5b). It is believed that Wearie-Phelan packing provides a minimal surface area for a given volume of cells/bubbles. The periodic domain (translation unit) of the Wearie-Phelan tessellation is shown in Fig. 5c.

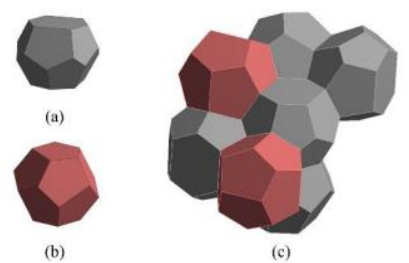

Figure 5. Wearie-Phelan packing

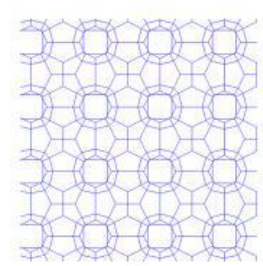

(a)

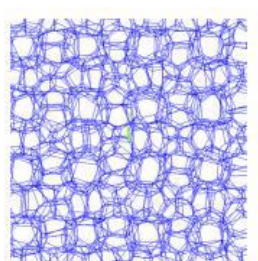

(b)

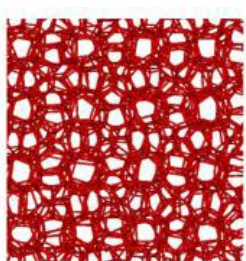

(c)
Figure 6. Mesoscale geometric modeling of aluminum foam: from structured frame to randomized solid model

It should be noted that the use of Wearie-Phelan idealization suggests a regular array of bubbles; however, real foams are random structures. To increase the realism of the modeling, therefore, a foam randomization mechanism is implemented. The overall algorithm for the geometric modeling can be described as follows: 1) generation of the representative volume element of aluminum foam with the specified dimensions based on Wearie-Phelan idealization [at 
this stage, the geometric model consists of multiple lines connected with each other at nodal points (see Fig. 6a)]; 2) randomization of the foam using node perturbation (see Fig. $6 \mathrm{~b}$ ); and 3) development of a solid geometric model using the randomized line-based geometry (see Fig. 6c).

The foam modeling algorithm has been implemented in the form of a script written in ANSYS Parametric Design Language. The SPH model of the foam-core sandwich panel is depicted in Fig. 7. It consists of approximately two million SPH particles. To represent thin ligaments of aluminum foam with a satisfactory accuracy, a smoothing length of $0.015 \mathrm{~mm} h \mathrm{as}$ been used. The model exploited the idea of quarter-symmetry. Although aluminum foam has a random structure and is not exactly symmetrical relative to any cutting plane, isotropy of its effective properties allows for such an assumption. The foam in the model is present only in the central region of the panel, where the most energetic fragments with the greatest damage potential will propagate. Additional lateral extension of the foam model was found to be impractical, as it was associated with high computational expenses.
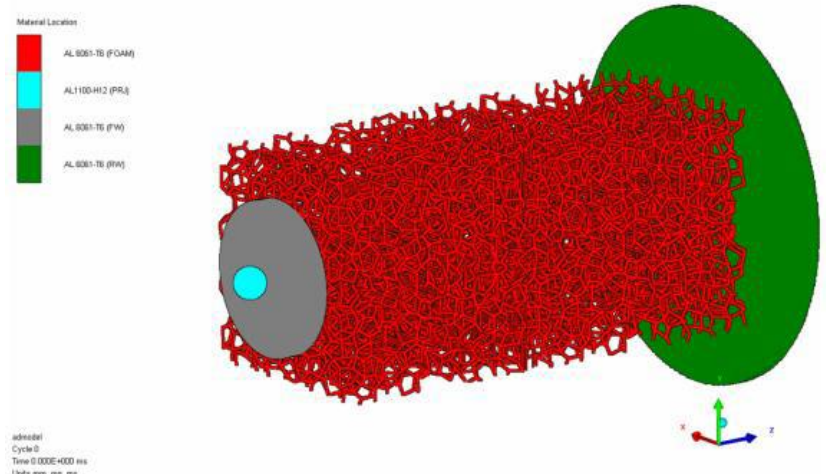

Figure 7. Numerical model of the open cell foam-core sandwich panel

Results of the conducted simulation are presented in Fig. 8, which demonstrates three consequent moments of time when the debris cloud propagates through the aluminum foam. It can be seen in the figure that multi-shock action of the foam effectively breaks up fragments of the projectile and the front bumper, converting them into a cloud of small, disperse particles. No perforation of the rear wall was detected during the numerical experiment.

In view of the novelty of the foam modeling approach (i.e., its mesoscale representation in HVI analysis), the numerical prediction was verified by means of a physical experiment. The experiment was carried out using a two-stage light gas gun at the HIT Dynamics, Ltd., impact testing facility (Canada). The tested panel consisted of two Al6061-T6 facesheets and 16mm-thick 3\% 40 ppi aluminum foam from ERG Aerospace. It should be noted that the tested panel had a slightly lower facesheet thickness $(0.4 \mathrm{~mm}$ instead of $0.5 \mathrm{~mm}$, as used in the numerical tests.
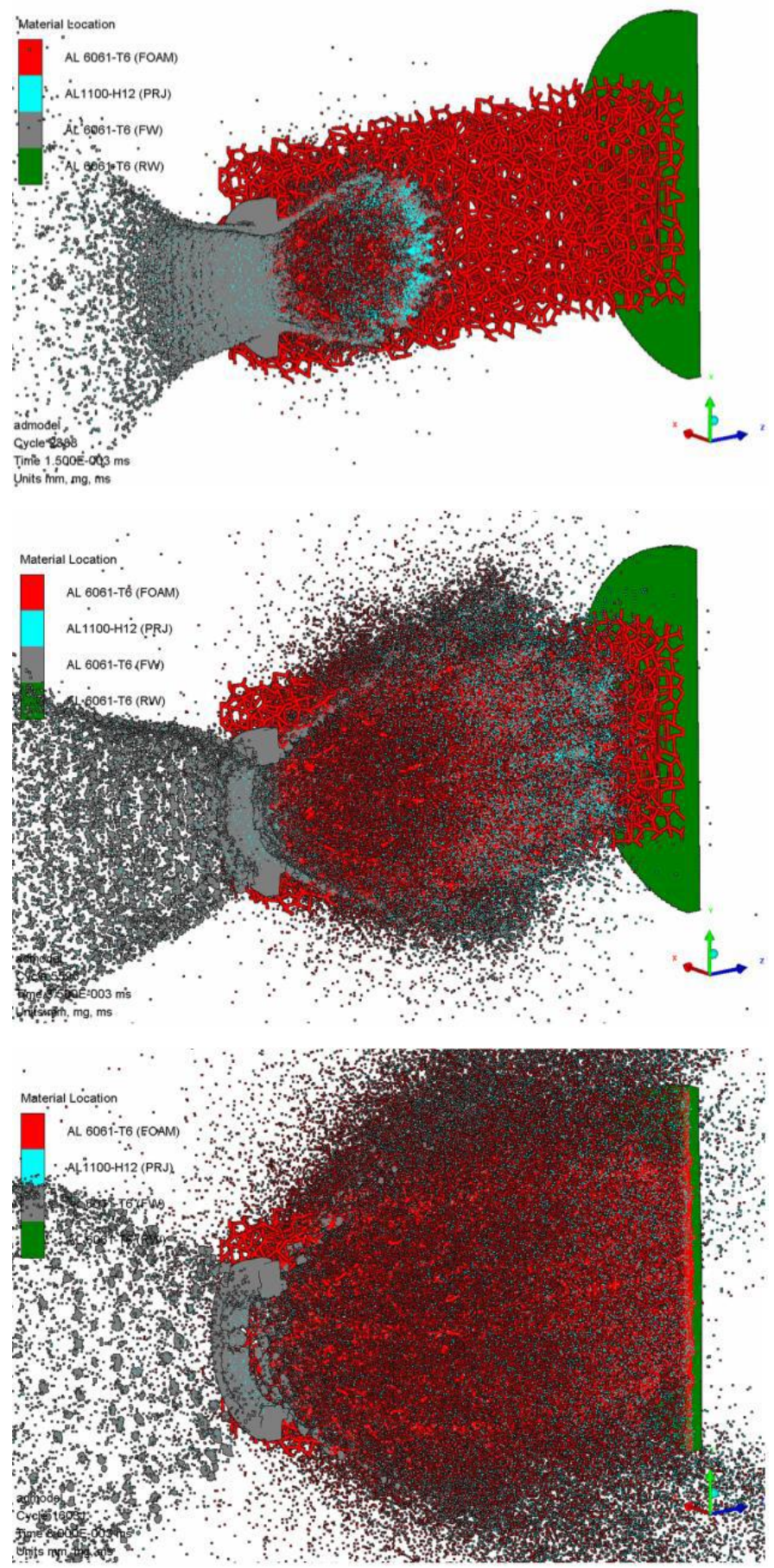

Figure 8. Results for the foam-core sandwich panel: a) $t=1.5$ $\mu s$; b) $t=3.5 \mu \mathrm{s}$; and c) $t=8.5 \mu \mathrm{s}$

The panel was impacted by a $1 \mathrm{~mm}$ aluminum projectile at $6.965 \mathrm{~km} / \mathrm{s}$, and the resulting damage to the panel is shown in Fig. 9. Rear-wall damage was represented by discoloration and barely noticeable bulging. No perforation of the rear wall was detected, which supported the results of the numerical simulation. 

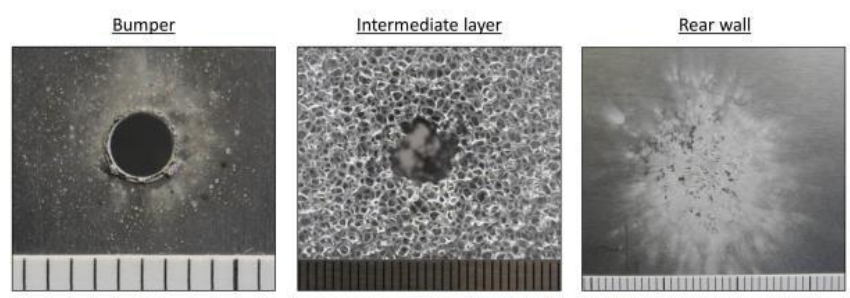

Figure 9. Experimental results for the foam-core sandwich panel
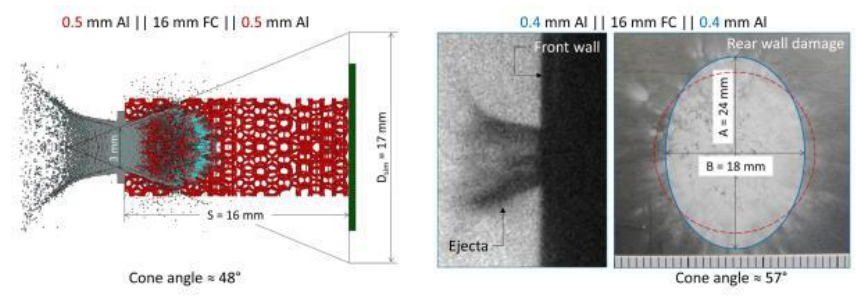

Figure 10. Shapes of ejecta clouds and cone angle measurements

Also, debris expansion angles (also referred to as "cone angles") were procured for both cases. For the model, this angle could be measured directly, as shown in Fig. 10, and this was found to be equal to $48 \mathrm{deg}$. To measure the experimental value of this angle, it was assumed that the debris cloud expanded within a cone, with one base having the diameter of the perforation hole in the front facesheet $(\sim 3 \mathrm{~mm})$ and the second base with the diameter of the damaged region on the rear wall. As the damaged area on the rear facesheet had a slightly elliptical shape (possibly because of the randomness of the foam; see Fig. 10), the corresponding cone base was represented in the calculations by a circle with the same area as the ellipse (indicated by the dashed line in Fig. 10). With this approximation, the debris expansion angle in the experiment was equal to $57 \mathrm{deg}$. The observed 9 deg difference was most likely the result of the different facesheet thicknesses of the modeled panel $(0.5 \mathrm{~mm})$ and the panel used in the experiment $(0.4 \mathrm{~mm})$. The model, therefore, was deemed to provide a good approximation of the physical phenomenon.

\section{MODELING OF FILAMENT-WOUND COMPOSITES}

Numerical techniques for the modeling of composite behavior under hypervelocity impact loading have been described in the literature $[5,7]$ and implemented in commercial software packages. These techniques are designed for the standard laminated composites. They are based on the homogenization of a composite laminate, which is represented in the modeling as a macroscopically homogeneous orthotropic media with effective properties equivalent to those of the real material.

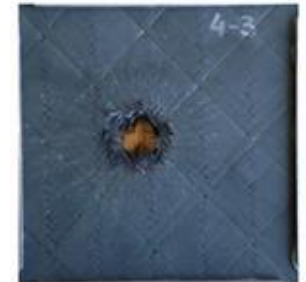

Front side

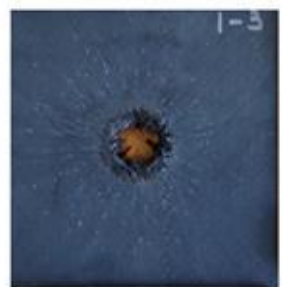

Front side

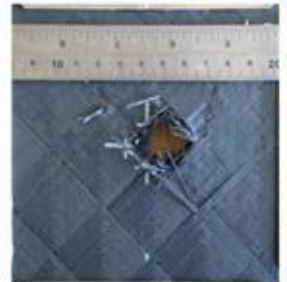

Rear side

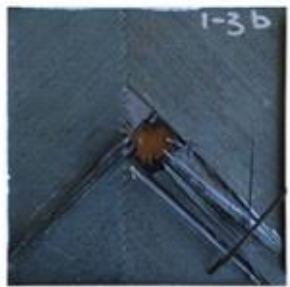

Rear side

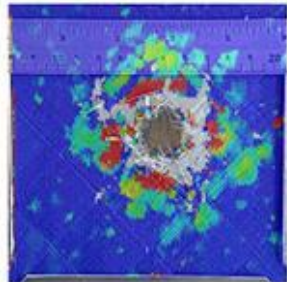

Ultrasound

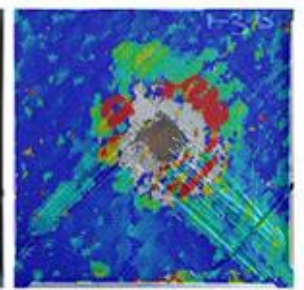

Ultrasound
Figure 11. HVI damage to a HDI composite (top) and a LDI composite material (bottom)

[impact conditions: $7 \mathrm{~km} / \mathrm{s} ; 4.76 \mathrm{~mm}$ aluminum projectile; 0.8 mm-thick aluminum bumper in front of the composite]

Conducted physical experiments revealed dependence of HVI damage of filament-wound composites on the particular winding pattern used in fabrication, as shown in Fig.11 In the case of pattern with the high density of interweaving (HDI) of filament bands, a specimen inspection revealed a "containment" of the impact damage within the region restricted by $2 \times 2$ weave units adjacent to the perforated hole. In case of low density of interweaving (LDI), the specimen damage propagates outwards along the direction of the fibers, especially in the bottom left and bottom right directions. Correspondingly, the homogenization-based techniques are inapplicable to this class of materials. Instead, in this study, a meso-scale modeling approach was used to represent the material structure in HVI simulations.

The meso-scale representation of the composite materials included explicit modeling of fiber-reinforced filament bands with ultrathin resin-rich regions between them. The following assumptions regarding the behavior of the materials of filament bands and resin-rich regions were made:

1. The material of a filament band is homogeneous, orthotropic, and linearly elastic until failure. The intra-band failure is assumed to be brittle, which is the case for most CFRP systems.

2. The material of the resin-rich regions is homogeneous, isotropic, and linearly elastic until failure. The linear elasticity assumption fits well for the thermoset-resin composites, as thermosets typically do not undergo a significant amount of plastic deformation prior to an initiation of failure.

3. Assumingly, the inter-band damage of the composite, such as delamination, is completely confined to the resin-rich region. The behavior in the resin-rich regions after a failure initiation is determined by such macro-scale parameters as fracture toughness, which allows for the consideration of both adhesive and cohesive types of inter-band fracture. 
4. Fracture toughness of thermoset-resin composites does not reveal strain-rate dependence, which is supported by several investigations by previous researchers [8 - 9].

As materials of filament bands and resin rich-regions are assumed to be linearly elastic until failure, their strength models only require the input of elastic constants. Pressure volume response was modeled using nonlinear EOS for composite filament bands, and the Gruneisen EOS for isotropic resin-rich layers. Failure within the filament bands and resin reach layers was modeled using a continuum damage mechanics model available in AUTODYN.

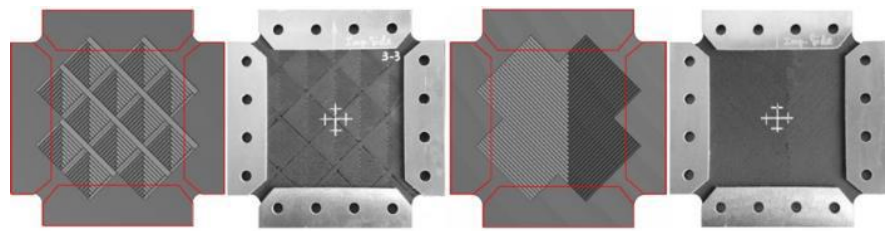

Figure 12. Geometrical modeling of HDI (left) and LDI (right) composites

The modeling approach employed in this study is based on simultaneous utilization of both SPH and FEM in each numerical simulation. The SPH method was used to represent the behavior of parts that exhibited fragmentation, namely aluminum projectile projectiles and bumpers. At the same time, composite panels with a meso-scale representation of filament winding patterns, which contained multiple interfaces between filament bands and resin-rich regions, were modeled using FEM in the Lagrangian formulation combined with the erosion mechanism. The use of erosion helped to avoid excessive mesh distortions and tangling at high deformations.

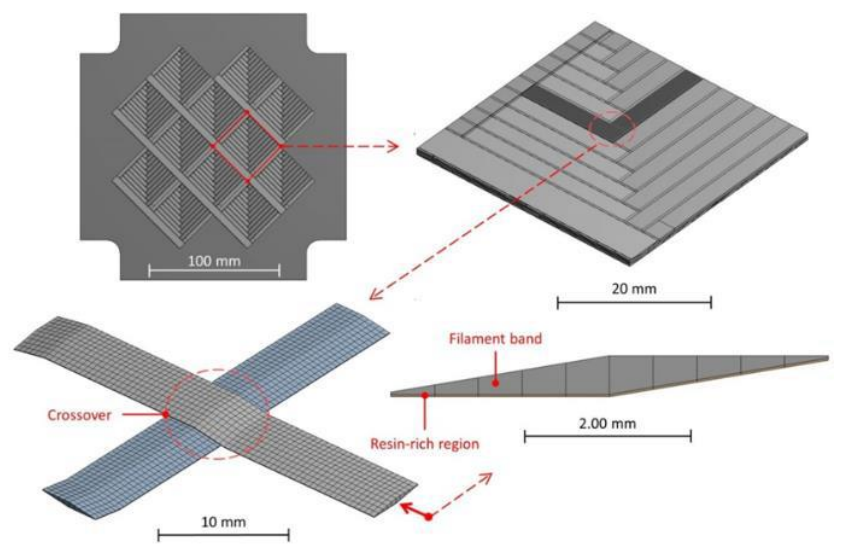

Figure 13. Meso-scale modeling of the filament-wound composite

The detailed meso-scale representation of filament winding was used only in the region of interest to minimize the computational cost. Around that region, the composite was represented by a homogeneous orthotropic material with effective properties equivalent to those of the real material. The geometrical models of the HDI and LDI panels compared with the real specimens are represented in Fig. 12.
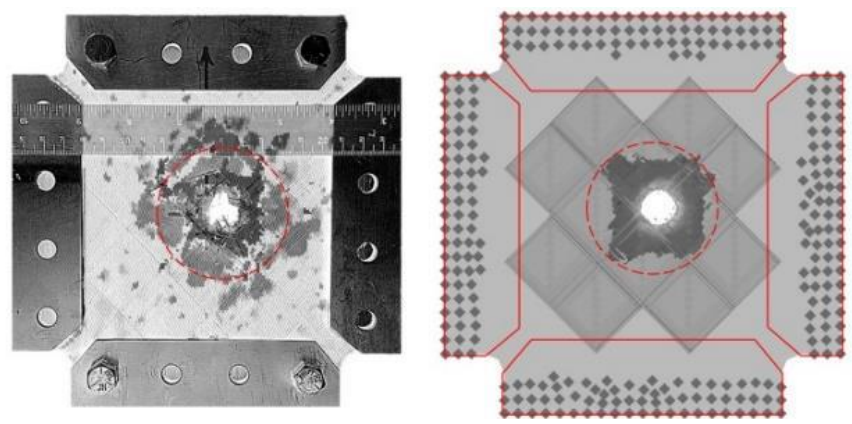

HDI: superposition of all damaged surfaces
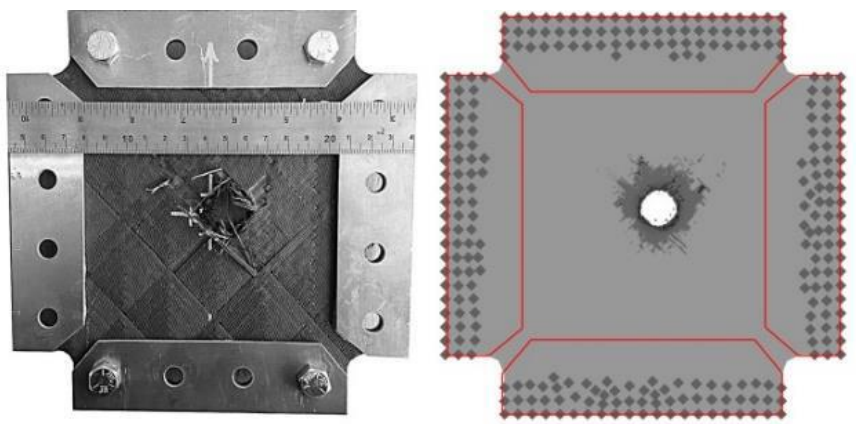

HDI: rear surface damage only
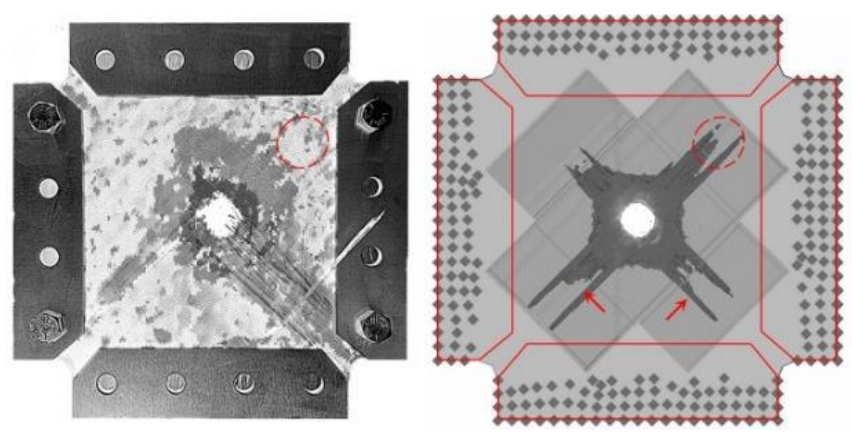

LDI: superposition of all damaged surfaces
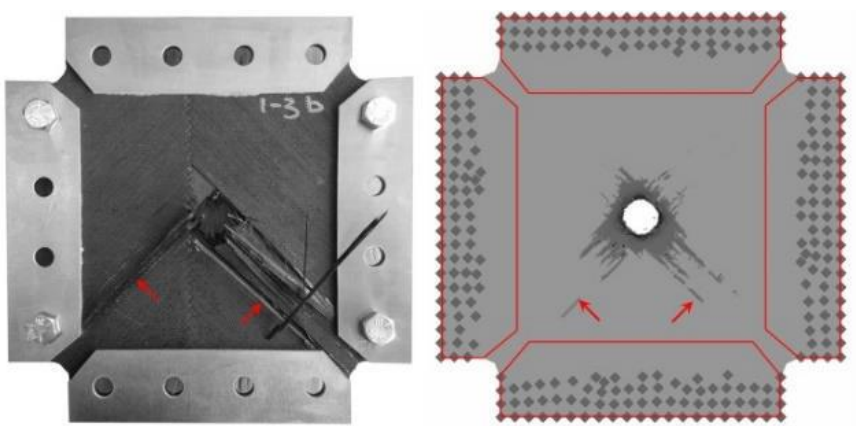

LDI: rear surface damage only

Figure 14. Composite damage: comparison of experimental results and numerical predictions

Fig. 13 represents the main features of the numerical models, including: a macro-scale appearance of the specimen model; a translational unit of the filament winding pattern model; a pair of interweaving filament bands; and a crosssection of a filament band with an adjacent resin-rich region. 
Voids at crossover points were represented explicitly in the meso-scale model.

Fig. 14 represents comparison of experimental and modeling results in terms of the damage to composite panels. It reveals the following:

- Simulations tend to somewhat underpredict the damaged area as compared to the damage detected by C-Scan. The exact reason for the underprediction is unknown, but may be attributed to the presence of a strain-rate dependence of the composite fracture toughness that was ignored in the modeling.

- For the HDI specimen, the simulation predicts a "containment" of the HVI damage within the region restricted by $2 \times 2$ HDI units adjacent to the perforated hole, which is the same damage pattern observed in the experiment.

- For the LDI specimen, the simulation predicts further propagation of the damage outwards along the fiber direction, which is also consistent with the experimental results. It should be noted, however, that the simulation also predicts the propagation of damage in the top-right corner of the specimen (encircled in Fig. 14) that was not observed on the C-Scan image.

- Damage patterns on the rear surface predicted by simulations for both HDI and LDI specimens are qualitatively in very good agreement with the experiment, as shown in Fig. 14.

\section{CONCLUSIONS}

For the foam-core sandwich panels, the meso-scale representation of the foam geometry is essential in HVI simulations, as it accounts for multi-shock interactions of the foam ligaments and hypervelocity fragment cloud propagating through the foam. The modelling approach considered in this study can be used in simulations estimating ballistic limit of foam core sandwich panels under hypervelocity impact.
Hypervelocity impact damage of a filament-wound composite is significantly dependent on the filament winding pattern used in its fabrication. In the presence of the winding pattern dependence, adequate simulation of HVI damage requires the utilization of a relatively high level of detail in representing a filament-wound composite. In this regard, the introduced meso-scale modeling approach was found to be suitable for capturing the main features of the HVI damage in the composites with different winding patterns. This approach can be used in the design of spacecraft filament-wound components, in order to determine the critical level of damage that is sustained due to orbital debris impacts.

\section{REFERENCES}

[1] Yasensky J., Christiansen E. (2007) Hypervelocity Impact Evaluation of Metal Foam Core Sandwich Structures. JSC63945.

[2] Ryan S., Hedman T., Christiansen E.L. (2010) Honeycomb vs. foam: Evaluating potential upgrades to ISS module shielding. Acta Astronautica 67, 818-825.

[3] Ryan S., Christiansen E.L. (2013) Hypervelocity impact testing of advanced materials and structures for micrometeoroid and orbital debris shielding. Acta Astronautica 83, 216-231.

[4] Yew C.H., Rodney B.K. (1987) A study of damage in composite panels produced by hypervelocity impact. Int J Impact Eng 5, 729-38.

[5] Wicklein M., Ryan S., White D.M., Clegg R.A. (2008) Hypervelocity impact on CFRP: testing, material modelling, and numerical simulation. Int J Impact Eng 35 (1), 1861-1869.

[6] Weaire, D., and Phelan, R. (1994) A counter-example to Kelvin's conjecture on minimal surfaces. Philosophical Magazine Letters 69 (2), 107-110.

[7] White D.M., Tylor E.A., Clegg R.A. (2003) Numerical simulation and experimental characterization of direct hypervelocity impact on spacecraft hybrid carbon fibre/Kevlar composite structure. Int J Impact Eng 29 (1), 779-790.

[8] Sun C.T., Han C. (2004) A method for testing interlaminar dynamic fracture toughness of polymeric composites. Compos Part B Eng 35(68), 647-55.

[9] Tsai J.L., Guo C., Sun C.T. (2001) Dynamic delamination fracture toughness in unidirectional polymeric composites. Compos Sci Technol $61(1), 87-94$. 